\title{
A new equilibrium for supramolecular chemists
}

\author{
Early-career researchers have been particularly impacted by the COVID-19 pandemic. To re-assemble the \\ supramolecular research community, the inaugural Women in Supramolecular Chemistry workshop promoted a \\ community-led approach to skills development, as Ruhee Dawood and Alyssa-Jennifer Avestro recount.
}

S upramolecular chemistry concerns the study of multi-component, often dynamic, assemblies whose emergent properties are greater than the sum of their individual molecular components. The same can be said of research communities. Collaboration, interdisciplinarity and the gathering of diverse talent, for example, are factors proven to generate the most meaningful scientific advances. They can also help drive positive systemic change in academia ${ }^{1}$. When the COVID-19 crisis brought laboratory-based activities and scientific meetings to an indefinite pause, there was a united concern across the scientific community about how this generation of early-career researchers would recover. Now, as COVID-19 cases begin to drop in some parts of the world, re-assembling in person is becoming more tangible and local research communities are tasked with establishing a new equilibrium.

It is in this context that the 1st Women in Supramolecular Chemistry (WISC) workshop was held in early September 2021. Over three days, it aimed to provide early-career scientists with a practical foundation in experimental techniques most commonly employed in supramolecular chemistry research. At a typical scientific conference, the emphasis of talks tends to be on highlighting major results and output. At the WISC workshop, however, expert speakers were instead encouraged to adopt more didactic approaches to promote knowledge exchange and practical skills development in the context of their world-leading research. Moreover, the workshop programme aimed to devote more than $75 \%$ of speaker time for its early-career delegates to shine.

The WISC network was launched in 2019 to unite all women, broadly defined ${ }^{2}$, across the field and develop support structures that promote their career progression and retention in academia. Don't let the moniker mislead you though - WISC events, discussion panels and online resources have always been open to supramolecular researchers of all gender identities, upholding the idea that fostering the best practices for equality should involve everyone and will ultimately benefit

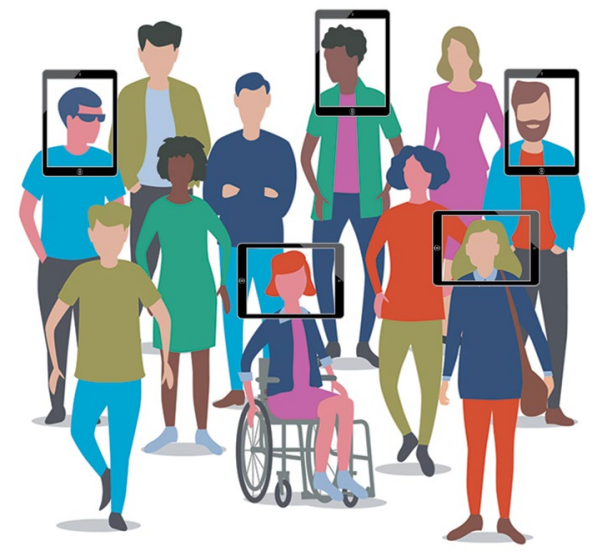

Credit: smartboy10/DigitalVision Vectors/Getty

everyone. WISC career and diversity panels have become a defining feature of the Royal Society of Chemistry's Macrocyclic and Supramolecular Chemistry (MASC) interest group meetings. Early-career meeting participants are invited to actively engage in policy development and conversations concerning equality, diversity and inclusion (EDI) in academia. More recently, WISC has played a major role in delivering the hugely successful virtual MASC (vMASC) seminar series, which has provided critical solace and unity for researchers coping with laboratory shutdowns and travel restrictions during the COVID-19 pandemic.

Continued disruptions caused by COVID-19 on travel plans and meeting logistics inspired WISC workshop co-organisers Claudia Caltagirone (University of Cagliari, Italy), Jennifer Hiscock (University of Kent, UK), Cally Haynes (University College London, UK) and Anna McConnell (University of Kiel, Germany) to adopt a hybrid format, allowing the event to remain accessible to remote participants without entirely sacrificing the opportunity to experience picturesque Cagliari, Sardinia, Italy, for those who could get there. Partnering with the vMASC seminar series also meant that the now hybrid WISC workshop and virtual poster session could be extended easily to accommodate over 200 virtual attendees from around the world (albeit most still attending from the UK and Europe.)

The pre-workshop poster session was hosted virtually a week before the workshop itself on Gather.Town - an interactive web-conferencing platform that facilitates more natural engagement between poster presenters and audience for immediate feedback compared with other virtual conferencing platforms. On designing their personal avatar, attendees were free to roam a custom-designed virtual space (in this case, a multi-room poster hall), interact with 36 posters 'hung' on display, and engage with other participant avatars (think Pokémon). In addition to conventional chatroom and video call facilities, the Gather.Town platform offered a satisfying simulation of the natural encounters associated with live poster sessions. For instance, participant avatars navigating within public 'earshot' of one another could overhear and easily dip in and out of conversations happening in real time. Meanwhile, unique functions to 'find' or 'follow' participants offered delightful novelties for socializing, whereas individually designated poster areas afforded presenters the attention of their audiences undisturbed. The nature of the platform meant attendees could personally manoeuver social encounters in the same manner as they would at a live networking event, engaging in as much - or as little - social interaction as they wished. And although holding the poster session one week ahead of the workshop seemed somewhat unconventional, there was good motive for doing so: the three poster prize winners would be invited as guest speakers on the final day to close the workshop.

The workshop itself featured a dense line-up of 46 virtual and in-person speakers organised across 10 loosely themed sessions over three days. The sessions were generally kicked off by plenary or invited talks from established leaders in the field to set a strong foundation for the research presentations to come - the majority of which were given by early-career researchers. Ivan Huc (Ludwig Maximilian University, Germany), for example, introduced foldamer chemistry by relating his group's investigations to derive function from organic molecules that 
fold and self-assemble into structures akin to those of proteins. As a result, they have achieved selective co-recognition of their synthetic helices with biomacromolecules ${ }^{3}$. Valeria Amendola (University of Pavia, Italy) presented a variety of spectroscopic techniques for quantifying the sensing capabilities of designer azacryptands ${ }^{4}$. Michaele Hardie (University of Leeds, $\mathrm{UK})$ related the most common pitfalls of crystallographic analysis before offering clever strategies to derive meaningful insights from nominally 'unpublishable' data $^{5}$. A day of thought-provoking science was topped off by a session led by Luca Prodi (University of Bologna, Italy) and Laura Rodriguez (University of Barcelona, Spain) who relayed their groups' research to develop dye-doped silica nanoparticles for nanomedicine ${ }^{6}$ and to elucidate the aggregation properties of gold- and platinum-containing organometallic complexes, respectively ${ }^{7}$. Day 1 ended with a dedicated flash talk session for in-person delegates to present their posters.

The second day offered a lighter programme, to ward off online fatigue and to also allow in-person delegates an opportunity to enjoy the sights of Cagliari. Jonathan Steed (Durham

University, UK) opened the day's scientific discussions by describing the hierarchical assembly processes of urea-based low-molecular-weight gelators ${ }^{8}$, which his group has used to selectively crystallize the active polymorphs of pharmaceuticals. Later, Hennie Valkenier (Université Libre de Bruxelles, Belgium) demonstrated how fluorescence spectroscopy could be used to monitor the activity of calixarenes as transmembrane ion transporters. Between these two sessions, a WISC panel discussion offered a welcome change of pace, "calling in" ${ }^{2}$ its community members to inform the next steps for WISC by reflecting on the EDI issues most relevant to them. Specifically, delegates were asked to consider the barriers hardest felt by women of any ethnicity and/ or members of other marginalized groups, and to suggest support structures that they would want to see implemented. An interactive web-based 'padlet' board was used to assemble feedback from in-person and virtual delegates. Managing childcare responsibilities, environments that inspire imposter syndrome to develop, and the burden of 'token diversity' were some of the most pressing challenges that delegates felt needed to be addressed. In the past, WISC has been proactive about gathering feedback from the community and developing well-defined support 'clusters' in response, such as for parents and carers, as well as for first-generation researchers (that is, the first in their families to attend university). Thus, additional WISC clusters (or other projects) may emerge as a result of this panel discussion.

The final day of the workshop featured an exciting line-up of international speakers. Sessions drew attention to metal-containing supramolecular systems such as coordination cages and metal-organic frameworks. Valérie Heitz (Université de Strasbourg, France) described her group's work to encapsulate guests and exact allosteric control of electron transfer within biomimetic metalloporphyrin cages $^{9}$. Meanwhile, Kay Severin (École Polytechnique Fédérale de Lausanne, Switzerland) enticed the audience with metallocage symmetry, detailing his group's studies to selectively achieve heteroleptic low-symmetry assemblies under thermodynamic control ${ }^{10}$. Ross Forgan (University of Glasgow, UK) then introduced different strategies to modulate the self-assembly and physical properties of metal-organic frameworks for potential applications ${ }^{11}$. Lastly, Luisa De Cola (University of Milano, Italy) demonstrated the role of confocal imaging microscopy to elucidate self-assembly processes involving luminescent $\mathrm{Pt}(\mathrm{II})$ complexes intended as bioimaging agents ${ }^{12}$.

The whole event closed with a celebration of the three poster prize winners selected the previous week. Samantha Bodman (Loughborough University, UK), Chelsea Wilson (University of Victoria, Canada) and Selina Hollstein (University of Ulm, Germany) each received prizes sponsored generously by Chemical Science, Chemical Communications and Fluorochem, respectively, and each delivered a guest talk on their research. The deliberate attention paid to early-career researchers has become a characteristic feature of WISC events that we hope will become more commonly practised at other scientific meetings. It was clear that attendees were appreciative of the manner in which the programme put the development, needs and achievements of early-career researchers clearly at centre stage. The didactic style of plenary and invited talks was also well-suited to practical advice that attendees could realistically take away and apply in their own research.

According to the post-workshop impact survey, which can be found on the WISC website, the blended in-person/virtual modality enabled over 250 workshop participants to attend - at least 2.5 times more participants than originally targeted, with $80 \%$ of all delegates tuning in virtually. Data from about 45 delegates also showed that they weren't necessarily studying or conducting research in the country they were born or raised in. This emphasises what early-career researchers are very aware of but societies are at risk of forgetting: that science is global. The WISC network is just one of several recent community-led initiatives that works to get this message across.

As for the future of scientific meetings in this (post-)COVID era? Tentatively unclear. Many research institutions are starting to transition back to the live seminar, yet for many of our peers and colleagues worldwide, the pandemic still makes in-person interactions difficult - though we hope for not much longer! As we have experienced with the 1st WISC workshop, the successful execution of a blended modality, alongside clever interactive platforms, can provide a satisfying surrogate even for ambitious multi-day scientific programmes. Beyond a pandemic, there are numerous other reasons to appreciate remote networking: for example health-related, due to caring commitments, and/or arising from financial or environmental considerations, to name but a few. Considering the bigger-picture goals of WISC and our academic research community, we sincerely hope that hybrid events are here to stay, improving and preserving diversity and inclusion in science - no matter what future challenges may next come our way.

Ruhee A. Dawood and

Alyssa-Jennifer Avestro $\bowtie$

Department of Chemistry, University of York, York, UK.

Twitter: @ajavestro

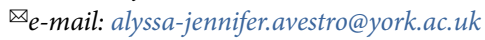

Published online: 22 November 2021 https://doi.org/10.1038/s41557-021-00849-1

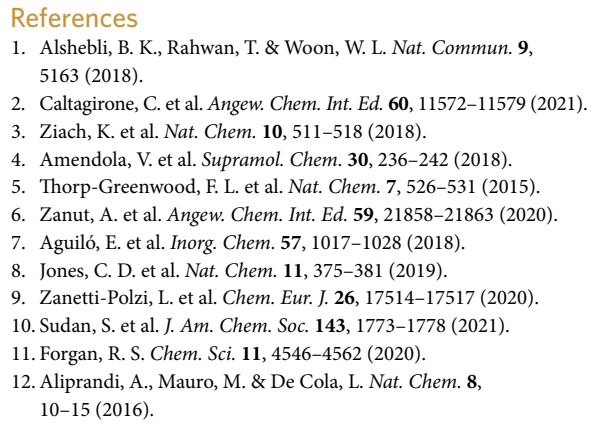

